\title{
Avaliação eletrocardiográfica de macacos-prego (Sapajus apella) sob contenção química com midazolam e propofol
}

\section{Electrocardiographic assessment of capuchin monkeys (Sapajus apella) under chemical contention with midazolam and propofol}

\author{
Luiz Guilherme Achcar Capriglione ${ }^{1^{*}}$; Grazielle Cristina Garcia Soresini²; \\ Taíse Fuchs ${ }^{1}$; Nathalia Trevelin Sant'Anna²; Ana Laura D'Ámico Fam³; \\ Claudia Turra Pimpão ${ }^{4}$; Ana Paula Sarraff-Lopes ${ }^{4}$
}

\begin{abstract}
Resumo
Estudos sobre eletrocardiograma (ECG) em Sapajus apella são restritos na literatura científica. Primatas não humanos podem ser sedados com benzodiazepínicos e após induzidos e mantidos anestesiados com propofol. O objetivo do presente estudo foi à descrição dos achados eletrocardiográficos em 18 macacos-prego (Sapajus apella) após sedação com midazolam e anestesia com propofol. A interpretação do eletrocardiograma foi realizada determinando-se os seguintes parâmetros: frequência $(\mathrm{FC})$ e ritmo cardíaco, eixo elétrico $\left(^{\circ}\right)$, onda $\mathrm{P}$, onda $\mathrm{R}$, onda $\mathrm{T}$, complexo $\mathrm{QRS}$, intervalo $\mathrm{P}-\mathrm{R}$, intervalo Q-T e segmento ST. A FC média foi de $198,4 \pm 22,9 \mathrm{bpm}$, a maioria dos macacos tinham ritmo sinusal normal, seguido de ritmo sinusal normal com marcapasso migratório. O eixo elétrico mais comumente observado manteve-se entre $60-90^{\circ}$. A administração intramuscular de midazolam, seguida de anestesia geral de curta ação com propofol não causou arritmias no traçado eletrocardiográfico e preservou a FC nos limites considerados fisiológicos para a espécie. Consequentemente, houve uma duração maior do intervalo Q-T comparado com estudos anteriores que utilizaram somente cetamina.

Palavras-chave: Sapajus apella, propofol, midazolam, eletrocardiograma
\end{abstract}

\begin{abstract}
Studies on electrocardiography in Sapajus apella are restricted in the scientific literature. Nonhuman primates can be sedated with benzodiazepine and after induced and maintained anesthetized with propofol. The objective of the present study was to description of the electrocardiographic findings in 18 capuchin monkeys (Sapajus apella) after sedation with midazolam and anesthesia with propofol. The interpretation of the ECG was performed by determining the following parameters: frequency (HR) and heart rhythm, electrical axis $\left(^{\circ}\right) \mathrm{P}$ wave, $\mathrm{R}$ wave, T wave, QRS complex, P-R interval, Q-T interval and ST segment. The HR mean was $198,4 \pm 22,9 \mathrm{bpm}$, the majority of monkeys had normal sinus rhythm, followed by normal sinus rhythm with wandering pacemaker. The most commonly observed electrical axis was between $60-90^{\circ}$. Intramuscular administration of midazolam, followed by a shortacting general anesthesia with propofol did not cause arrhythmias in electrocardiographic tracing and preserved the $\mathrm{FC}$ in the limits considered physiological for to specie. Consequently, there was a longer

\footnotetext{
${ }^{1}$ Pesquisadores em Anestesiologia Veterinária, Pontifícia Universidade Católica do Paraná, PUCPR, São José dos Pinhais, PR. E-mail: luizcapriglione@hotmail.com; taisefuchs@hotmail.com

${ }^{2}$ Pesquisadoras em Clínica e Cirurgia de Animais Selvagens, PUCPR, Centro de Triagem de Animais Silvestres, CETAS, Tijucas do Sul, PR. E-mail: grasoresini@yahoo.com.br; nathresant@hotmail.com

${ }^{3}$ Pesquisadora em Patologia Veterinária, PUCPR, São José dos Pinhais, PR. E-mail: anadamico@gmail.com

${ }^{4}$ Prof ${ }^{\text {as }}$ da PUCPR, São José dos Pinhais, PR. E-mail: claudia.pimpao@pucpr.br; ana.sarraff@pucpr.br

* Autor para correspondência
} 
duration of the Q-T interval compared with previous studies that utilized only ketamine.

Key words: Sapajus apella, propofol, midazolam, electrocardiogram

\section{Introdução}

O macaco-prego (Sapajus apella), espécie arborícola diurna de primata do Novo Mundo, distribui-se geograficamente em vários países da América do Sul (VERONA; PISSINATTI, 2007). $\mathrm{O}$ desmatamento progressivo das florestas e consequentemente a destruição do habitat natural destes animais faz com que migrem para outras regiões, tornando-os vulneráveis à caça predatória e a retirada da natureza, o que leva a um grande número de exemplares em cativeiro. O CETAS (Centro de Triagem de Animais Silvestres) de Tijucas do Sul/ PR recebe frequentemente indivíduos desta espécie, provenientes de apreensões realizadas por órgãos de fiscalização ou entrega voluntária.

O Eletrocardiograma (ECG) é um exame simples e essencial para determinar a frequência e o ritmo cardíaco. Auxilia no diagnostico das arritmias cardíacas e distúrbios de condução, bem como no seu direcionamento terapêutico. Constantemente macacos-prego necessitam de anestesia para realização de exames ou procedimentos cirúrgicos, sendo fundamental o monitoramento da função cardiorrespiratória durante a anestesia. $\mathrm{O}$ índice de mortalidade de pequenos mamíferos silvestres é maior durante anestesia ou sedação quando comparado aos animais domésticos, como cão, gato e coelho, sendo os cuidados ao anestesiar estas espécies mais intensos (BRODBELT et al., 2008). Outro fator a ser considerado é o fato de a espécie ser frequentemente utilizada em protocolos experimentais envolvendo infecções por Trypanosoma cruzi, que mimetizam a fisiopatologia da doença de Chagas em humanos, a qual resulta em diversas arritmias cardíacas (FALASCA et al., 1991). Portanto, o conhecimento do padrão eletrocardiográfico normal nesta espécie faz-se necessário.

O macaco-prego pode ser capturado utilizandose puçá e contido fisicamente aproximando e mantendo os dois cotovelos juntos às costas. Os agentes benzodiazepínicos podem ser usados por via parenteral ou oral previamente a anestesia, proporcionando sedação, relaxamento muscular e efeito anticonvulsivante (VERONA; PISSINATTI, 2007). Em mamíferos, a administração de midazolam produz mínimos efeitos sobre a função cardiopulmonar (LEMKE, 2007).

O propofol é classificado como um derivado alquifenol sedativo-hipnótico intravenoso que ganhou grande popularidade na prática veterinária e humana devido as suas propriedades desejáveis na anestesia injetável (FOWLER et al., 2001). O propofol pode ser usado como agente indutor e na manutenção da anestesia de primatas não humanos (FOWLER et al., 2001, FLECKNELL; RICHARDSON; POPOVIC, 2007). Segundo um estudo realizado em macacos da espécie Macaca mulatta, demonstrou que o tempo de recuperação anestésica após contenção química utilizando propofol $(6 \mathrm{mg} / \mathrm{kg} / \mathrm{IV})$ foi de $22,5 \pm 3,2$ minutos, ligeiramente inferior quando comparado à associação cetamina $(8 \mathrm{mg} / \mathrm{kg} / \mathrm{IM})$ midazolam $(0,2 \mathrm{mg} / \mathrm{kg} / \mathrm{IM})$, que durou 41,2 $\pm 5,9$ minutos. O propofol tem sido amplamente utilizado em protocolos de anestesia intravenosa, sendo seu uso muito difundido na Medicina Veterinária e humana (SUAREZ et al., 2012, YOO et al., 2012).

Um importante fator em relação ao ECG em espécies exóticas, é que muitos padrões de normalidade não foram estabelecidos em função do protocolo anestésico utilizado (FELIPPE, 2007, LARSSON et al., 2012). Em macacosprego, padrões eletrocardiográficos referentes à ECG encontram-se restritos as avaliações sob anestesia com cetamina (GONDER; GARD; LOTT, 1980, LARSSON et al., 2012). Santana et al. (2008) investigaram a associação tiletaminazolazepam e cetamina-xilazina, contudo estudos 
descritivos sobre as alterações eletrocardiográficas em macacos-prego sedados com midazolam e anestesiados com propofol não foram encontrados.

Devido à escassa literatura sobre o assunto, foi realizado um estudo cujo objetivo foi determinar o padrão eletrocardiográfico em 18 macacosprego (Sapajus apella), sedados com midazolam e anestesiados com propofol.

\section{Material e Métodos}

Todos os procedimentos foram realizados após aprovação do protocolo experimental pelo Comitê de Ética com Uso de Animais (CEUA) da Pontifícia Universidade Católica do Paraná (PUCPR), parecer 443.

Foram selecionados 18 (dezoito) exemplares de macaco-prego (Sapajus apella) adultos, sendo 12 (doze) machos e 6 (seis) fêmeas, pesando entre 1,2 e 4,5 kg, provenientes do Centro de Triagem de Animais Silvestres (CETAS PUCPR/IBAMA), situado no município de Tijucas do Sul/PR, aonde eram mantidos em recintos de $9 \mathrm{~m}^{2}$. A dieta oferecida diariamente era composta de frutas variadas, verduras, alimento extrusado para primatas não humanos (Alcon Club Monkey Cookies ${ }^{\circledR}$, Alcon Pet, Camburiú, Santa Catarina, Brasil) e fonte proteica oferecida duas a três vezes por semana (ovo cozido, carne com carbonato de cálcio ou camundongo). Antes do início do estudo foram realizados testes sorológicos para detecção de vírus rábico em todos os animais, sendo o resultado negativo.

Os macacos foram considerados saudáveis conforme exame clínico, hematológico e bioquímico sérico, considerando função hepática e renal. Os valores hematológicos e bioquímicos séricos foram consultados dos estudos de Larsson et al. (1997, 1999).

Os macacos foram submetidos a jejum hídrico e alimentar de 12 horas. Após serem capturados no recinto com auxílio de um puçá de rede e contidos fisicamente, foi administrado $1 \mathrm{mg} / \mathrm{kg}$ de midazolam
(Midazolam 0,5\%, Dormonid $\AA$, Produtos Roche Químicos e Farmacêuticos S.A., Rio de Janeiro, Brasil) por via intramuscular. Aguardou-se 10 minutos e em seguida, foi realizada tricotomia sobre a veia safena, e nos respectivos locais que os eletrodos foram fixados. A veia safena foi acessada com um cateter número 22 (Gauge), para administração de solução de Ringer com Lactato na taxa de $20 \mathrm{~mL} / \mathrm{kg} /$ hora e para a administração de propofol(Propofol 1\%, Fresofol®, Fresenius Kabi AB, Uppsala, Suécia) 10 $\mathrm{mg} / \mathrm{kg}$, em aproximadamente 30 segundos, conforme protocolo proposto por Flecknell, Richardson e Popovic (2007). Imediatamente após administração do propofol, foi realizada a intubação orotraqueal com sonda endotraqueal de silicone, com cuff e diâmetro de 2,5 mm. Os macacos foram mantidos em respiração espontânea em um sistema respiratório aberto com ambu pediátrico e fluxo de oxigênio em $200 \mathrm{~mL} / \mathrm{kg} / \mathrm{min}$. Após intubação orotraqueal, 1/3 da dose inicial do propofol foi novamente administrado para inibir movimentos involuntários causados pelo reflexo de tosse. Imediatamente após a realização da intubação orotraqueal foi realizado o ECG que durou aproximadamente três minutos.

Para realização do exame de ECG os animais foram posicionados em decúbito lateral direito, com os membros paralelos e perpendiculares ao eixo longo do corpo, sobre um colchão de borracha a fim de se evitar interferências. Utilizou-se eletrocardiógrafo convencional (Eletrocardiógrafo, Ecafix ${ }^{\circledR}$, ECG6, São Paulo, Brasil) e os cabos foram conectados da seguinte forma: o vermelho ao membro torácico direito, o amarelo ao torácico esquerdo, o preto ao pélvico direito e o verde ao pélvico esquerdo. Previamente a colocação dos eletrodos a pele foi umedecida com álcool. Os eletrodos foram posicionados acima da articulação úmero-rádio-ulnar e fêmoro-tíbio-patelar através de conectores metálicos do tipo "jacaré”. O registro eletrocardiográfico foi realizado na velocidade de $50 \mathrm{~mm} /$ segundo, sensibilidade $\mathrm{N}(1 \mathrm{~cm}=1 \mathrm{mV})$ nas derivações DI, DII, DIII, aVR, aVL e aVF. Em seguida foi realizada a interpretação do 
traçado eletrocardiográfico em derivação DII, determinando-se frequência (FC) e ritmo cardíacos, onda $\mathrm{P}$, intervalo $\mathrm{P}-\mathrm{R}$, onda $\mathrm{R}$, complexo $\mathrm{QRS}$, onda $\mathrm{T}$, intervalo Q-T, segmento ST e eixo elétrico $\left(^{\circ}\right)$ mediante tabela de eixos (DI/DIII). Os dados foram apresentados sob a forma individual, valor máximo e valor mínimo, média e desvio padrão.

Após o exame, os animais foram extubados após o retorno do reflexo de deglutição, e em seguida alocados nas gaiolas de contenção, sendo encaminhados para o recinto novamente após completa recuperação anestésica.

\section{Resultados}

As médias e variáveis de dispersão dos parâmetros do ECG dos 18 exemplares estão apresentados na tabela 1 . O ritmo sinusal normal foi encontrado em $88,8 \%$ (16/18) enquanto que o ritmo sinusal normal com marcapasso migratório foi presenciado em $11,1 \%(2 / 18)$. O eixo elétrico ficou entre $0-104^{\circ}$ em $100 \%$ dos macacos (18/18). Destes, dois macacos 11,1\% (2/18) obtiveram eixo elétrico entre $90-120^{\circ}, 10$ macacos 55,5\% (10/18) entre $60-90^{\circ}$, um macaco $5,5 \%(1 / 18)$ em $60^{\circ}$, três macacos $16,6 \%(3 / 18)$ entre $30-60^{\circ}$, um macaco $5,5 \%(1 / 18)$ em $30^{\circ}$ e um macaco $5,5 \%(1 / 18)$ em $0^{\circ}$. Os parâmetros individuais dos animais deste estudo de acordo com o sexo estão apresentados nas tabelas 2 e 3 , respectivamente. O complexo QRS foi positivo nas derivações DI, DII, DIII e aVF (figura 1). As ondas Q e S demonstraram-se relativamente pequenas e estreitas em derivação DII (figura 2).

Tabela 1. Médias e variáveis de dispersão dos parâmetros do ECG dos 18 macacos-prego (Sapajus apella) sedados com midazolam $(1 \mathrm{mg} / \mathrm{kg} / \mathrm{IM})$ e anestesiados com propofol $(10 \mathrm{mg} / \mathrm{kg} / \mathrm{IV})$. Parâmetros registrados na derivação DII, velocidade de $50 \mathrm{~mm} / \mathrm{s}$ e modo $\mathrm{N}$.

\begin{tabular}{ll}
\hline Parâmetros & $\begin{array}{l}\text { SAPAJUS APELLA } \\
\text { (média, desvio padrão, valor mínimo e máximo) }\end{array}$ \\
\hline FC (bpm) & $198,4 \pm 22,9(157$ a 230$)$ \\
P (seg/mV) & $0,04 \pm 0,007(0,03$ a 0,05$) / 0,13 \pm 0,04(0,1$ a 0,2$)$ \\
P-R (seg) & $0,08 \pm 0,01(0,06$ a 0,11$)$ \\
QRS (seg/mV) & $0,04 \pm 0,01(0,02$ a 0,06$) / 0,70 \pm 0,4(0,2$ a 1,7$)$ \\
ST (\%) & $\begin{array}{l}\text { Normal }(55,6 \% ; 10 \text { macacos }) \\
\text { Elevação } 0,1 \mathrm{mV}(27,8 \% ; 5 \text { macacos })\end{array}$ \\
Q-T (seg) & Depressão $0,1 \mathrm{mV}(16,7 \% ; 3$ macacos $)$ \\
T (polaridade positiva, negativa ou bifásica) & $0,17 \pm 0,03(0,08$ a 0,24$)$ \\
& Positiva $(77,8 \% ; 14$ macacos $)$ \\
Eixo elétrico (DI/DIII) & Negativa $(22,2 \% ; 4$ macacos $)$ \\
\hline
\end{tabular}

Siglas: ECG (eletrocardiograma); bpm (batimentos por minuto); FC (frequência cardíaca); P (onda P); P-R (intervalo PR); QRS (complexo QRS); R (onda R); T (onda T); Q-T (intervalo QT); ST (segmento ST); milivolts (mV); segundos (seg); pol (polaridade); ELE 0,1 (elevação de 0,1mV); DEP 0,1 (depressão de 0,1mV); N (normal); S (desvio padrão).

Fonte: Elaboração dos autores. 
Tabela 2. Variáveis do ECG individual dos 12 macacos-prego (Sapajus apella) machos, sedados com midazolam $(1 \mathrm{mg} / \mathrm{kg} / \mathrm{IM})$ e anestesiados com propofol $(10 \mathrm{mg} / \mathrm{kg} / \mathrm{IV})$. Parâmetros registrados na derivação DII, velocidade de 50 $\mathrm{mm} / \mathrm{s}$ e modo $\mathrm{N}$.

\begin{tabular}{|c|c|c|c|c|c|c|c|c|c|c|}
\hline $\begin{array}{l}\text { Sapajus } \\
\text { Apella }\end{array}$ & $\begin{array}{c}\text { FC } \\
(\mathrm{bpm})\end{array}$ & $\begin{array}{c}\text { P } \\
\text { (seg) }\end{array}$ & $\begin{array}{l}P \\
(\mathrm{mV})\end{array}$ & $\begin{array}{l}\text { P-R } \\
\text { (seg) }\end{array}$ & $\begin{array}{l}\text { QRS } \\
\text { (seg) }\end{array}$ & $\begin{array}{c}R \\
(\mathrm{mV}) \\
\end{array}$ & $\begin{array}{l}T \\
\text { (pol) }\end{array}$ & $\begin{array}{l}\text { Q-T } \\
\text { (seg) }\end{array}$ & ST & $\begin{array}{l}\text { Eixo } \\
\text { DI/DII( }\left(^{\circ}\right)\end{array}$ \\
\hline & 181,8 & 0,04 & 0,15 & 0,08 & 0,02 & 0,35 & + & 0,18 & $\mathrm{~N}$ & 74 \\
\hline & 200 & 0,04 & 0,1 & 0,08 & 0,02 & 0,4 & + & 0,18 & ELE 0,1 & 60 \\
\hline & 166,6 & 0,05 & 0,15 & 0,07 & 0,06 & 0,45 & + & 0,18 & $\mathrm{~N}$ & 78 \\
\hline & 181,8 & 0,04 & 0,15 & 0,09 & 0,02 & 1,0 & + & 0,2 & ELE 0,1 & 66 \\
\hline & 222,2 & 0,04 & 0,1 & 0,07 & 0,02 & 0,3 & + & 0,08 & $\mathrm{~N}$ & 101 \\
\hline & 200 & 0,04 & 0,1 & 0,08 & 0,03 & 1,0 & + & 0,16 & $\mathrm{~N}$ & 51 \\
\hline & 176,4 & 0,05 & 0,1 & 0,09 & 0,05 & 0,55 & + & 0,2 & ELE 0,1 & 104 \\
\hline & 214,2 & 0,03 & 0,1 & 0,07 & 0,05 & 0,95 & + & 0,18 & $\mathrm{~N}$ & 81 \\
\hline & 214,2 & 0,04 & 0,11 & 0,08 & 0,04 & 1,5 & + & 0,16 & $\mathrm{~N}$ & 73 \\
\hline & 187,5 & 0,05 & 0,2 & 0,09 & 0,06 & 0,5 & - & 0,16 & DEP 0,1 & 71 \\
\hline & 193,5 & 0,03 & 0,2 & 0,08 & 0,04 & 0,65 & + & 0,2 & $\mathrm{~N}$ & 84 \\
\hline & 200 & 0,03 & 0,2 & 0,08 & 0,04 & 1,7 & - & 0,16 & DEP 0,1 & 75 \\
\hline media & 194,8 & 0,04 & 0,13 & 0,08 & 0,03 & 0,77 & & 0,17 & & 76,5 \\
\hline $\mathrm{S}( \pm)$ & 16,8 & 0,007 & 0,04 & 0,007 & 0,01 & 0,45 & & 0,03 & & 15,1 \\
\hline
\end{tabular}

Siglas: ECG (eletrocardiograma); bpm (batimentos por minuto); FC (frequência cardíaca); P (onda P); P-R (intervalo PR); QRS (complexo QRS); R (onda R); T (onda T); Q-T (intervalo QT); ST (segmento ST); milivolts (mV); segundos (seg); pol (polaridade); ELE 0,1 (elevação de 0,1mV); DEP 0,1 (depressão de 0,1mV); $\mathrm{N}$ (normal); $\mathrm{S}$ (desvio padrão).

Fonte: Elaboração dos autores.

Tabela 3. Variáveis do ECG individual dos seis macacos-prego (Sapajus apella) fêmeas, sedados com midazolam $(1 \mathrm{mg} / \mathrm{kg} / \mathrm{IM})$ e anestesiados com propofol $(10 \mathrm{mg} / \mathrm{kg} / \mathrm{IV})$. Parâmetros registrados na derivação DII, velocidade de 50 $\mathrm{mm} / \mathrm{s}$ e modo $\mathrm{N}$.

\begin{tabular}{lllllllllll}
\hline $\begin{array}{l}\text { Sapajus } \\
\text { Apella }\end{array}$ & $\begin{array}{c}\text { FC } \\
(\mathbf{b p m})\end{array}$ & $\begin{array}{c}\mathbf{P} \\
(\mathbf{s e g})\end{array}$ & $\begin{array}{c}\mathbf{P} \\
(\mathbf{m V})\end{array}$ & $\begin{array}{l}\text { P-R } \\
\mathbf{( s e g})\end{array}$ & $\begin{array}{l}\text { QRS } \\
(\mathbf{s e g})\end{array}$ & $\begin{array}{c}\mathbf{R} \\
(\mathbf{m V})\end{array}$ & $\mathbf{T}$ & $\begin{array}{l}\text { Q-T } \\
(\mathbf{s e g})\end{array}$ & $\mathbf{S T}$ & $\begin{array}{l}\text { Eixo } \\
\mathbf{D I} / \mathbf{D I I}\left({ }^{\circ}\right)\end{array}$ \\
\hline & 171,4 & 0,03 & 0,1 & 0,06 & 0,05 & 0,6 & + & 0,24 & $\mathrm{~N}$ & 65 \\
& 230 & 0,03 & 0,1 & 0,1 & 0,04 & 0,2 & + & 0,18 & ELE 0,1 & 30 \\
& 157,8 & 0,05 & 0,15 & 0,11 & 0,06 & 0,85 & + & 0,2 & DEP 0,1 & 58 \\
& 230,7 & 0,05 & 0,15 & 0,09 & 0,06 & 0,6 & + & 0,14 & $\mathrm{~N}$ & 67 \\
& 214 & 0,04 & 0,2 & 0,1 & 0,03 & 0,35 & + & 0,1 & $\mathrm{~N}$ & 0 \\
& 230 & 0,04 & 0,1 & 0,08 & 0,05 & 0,8 & - & 0,22 & ELE 0,1 & 56 \\
med & 205,6 & 0,04 & 0,13 & 0,09 & 0,04 & 0,56 & & 0,18 & & 46 \\
$\mathrm{~S}( \pm)$ & 32,6 & 0,008 & 0,04 & 0,01 & 0,01 & 0,25 & & 0,05 & & 26,1 \\
\hline
\end{tabular}

Siglas: ECG (eletrocardiograma); bpm (batimentos por minuto); FC (frequência cardíaca); P (onda P); P-R (intervalo PR); QRS (complexo QRS); R (onda R); T (onda T); Q-T (intervalo QT); ST (segmento ST); milivolts (mV); segundos (seg); pol (polaridade); ELE 0,1 (elevação de 0,1mV); DEP 0,1 (depressão de 0,1mV); N (normal); S (desvio padrão).

Fonte: Elaboração dos autores. 
Figura 1. Registro do eletrocardiograma de um macaco-prego (Sapajus apella) clinicamente normal sedado com midazolam $(1 \mathrm{mg} / \mathrm{kg} / \mathrm{IM})$ e anestesiado com propofol $(10 \mathrm{mg} / \mathrm{kg} / \mathrm{IV})$. Velocidade do registro: $50 \mathrm{~mm} / \mathrm{s}, 1 \mathrm{~cm}=1 \mathrm{mV}$.

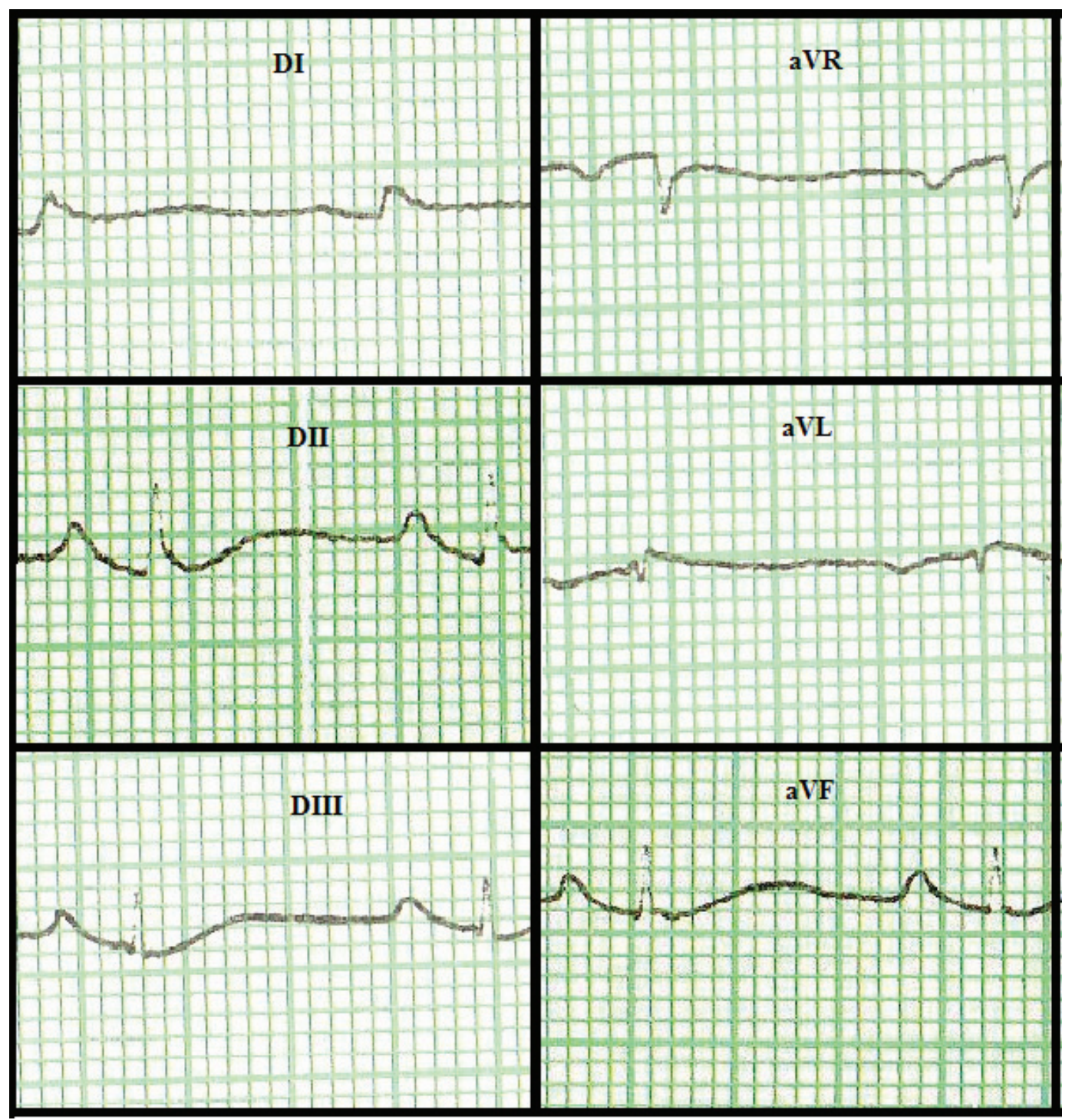

Fonte: Elaboração dos autores.

Figura 2. Registro do eletrocardiograma em derivação DII de um macaco-prego (Sapajus apella) clinicamente normal sedado com midazolam ( $1 \mathrm{mg} / \mathrm{kg} / \mathrm{IM})$ e anestesiado com propofol $(10 \mathrm{mg} / \mathrm{kg} / \mathrm{IV})$ apresentando ritmo sinusal normal. Velocidade do registro: $50 \mathrm{~mm} / \mathrm{s}, 1 \mathrm{~cm}=1 \mathrm{mV}$.

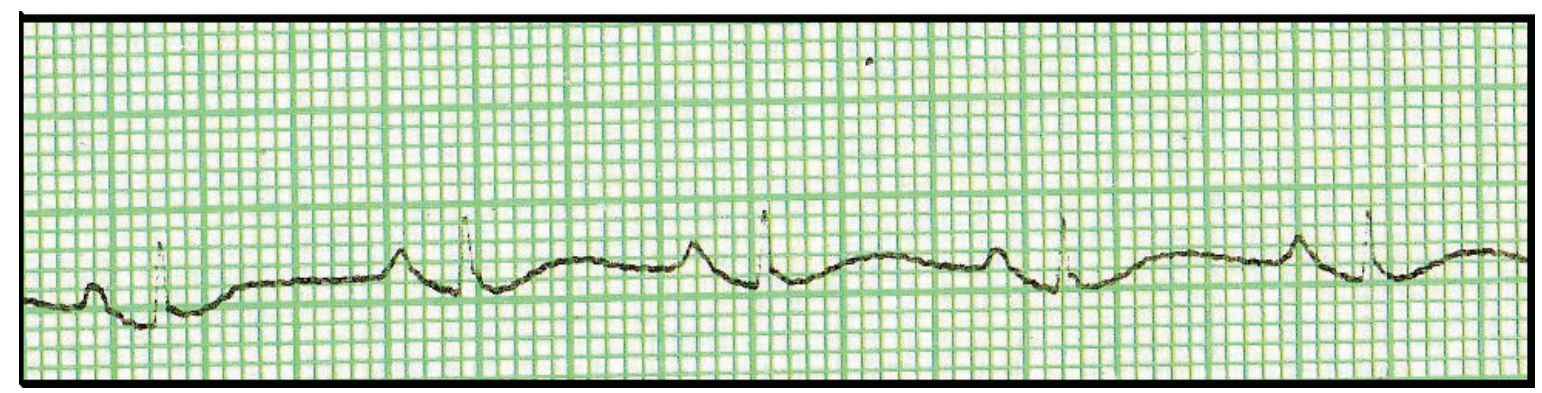

Fonte: Elaboração dos autores. 


\section{Discussão}

No presente estudo, foi investigada a ação do propofol sobre o ECG em macacos-prego. Primeiramente, este anestésico demonstrou ser seguro ao ser administrado na dose estudada, não ocorrendo apneia ou depressão respiratória significativa. Segundo, os macacos foram observados até sua completa recuperação, demonstrando uma recuperação rápida e tranquila, sem sinais de excitação do sistema nervoso central. Finalmente, o ritmo sinusal foi predominante no ECG, sem apresentar qualquer tipo de arritmia cardíaca durante o exame, concordando com Branson (2007) que relata que o propofol não é inerentemente arritmogênico e com Muir (2007) que cita que arritmias são raramente observadas com a administração de midazolam como agente pré-anestésico. A principal desvantagem do uso do propofol em relação aos anestésicos dissociativos é que obrigatoriamente é necessário um acesso venoso para sua administração.

$\mathrm{O}$ presente estudo sugere diferenças nos parâmetros eletrocardiográficos em macacos-prego de acordo com a contenção química utilizada. As arritmias cardíacas mais comuns podem ocorrer como resultado da dissociação celular devido ao número diminuído de conexões celulares efetivas, causadas por fibrose, isquemia ou efeitos de drogas (MUIR, 2007). Drogas anestésicas produzem marcadas mudanças nas propriedades eletrofisiológicas passivas e na atividade celular cardíaca resultando no desenvolvimento de arritmias cardíacas (MUIR, 2007). Neste estudo observamos que a contenção química com midazolam-propofol apresentou menor potencial de alterações na FC em relação aos valores fisiológicos desta espécie, que varia entre 165 e 225 bpm (VERONA; PISSINATTI, 2007). $\mathrm{O}$ que não ocorre com os protocolos que utilizaram somente cetamina (GONDER; GARD; LOTT, 1980, LARSSON et al., 2012), aonde foi observado taquicardia sinusal e com os agentes $\alpha 2$-agonistas que causam bradicardia severa nesta espécie (SANTANA et al., 2008). Isto ocorre pelo fato dos anestésicos dissociativos estimularem o sistema cardiovascular de forma indireta, incluindo efeitos simpatomiméticos e consequentemente elevando a FC (LIN, 2007). Por outro lado, a xilazina inibe a liberação de noradrenalina e consequentemente altera a atividade inotrópica, cronotrópica e eletrofisiológica (SANTANA et al., 2008). Potenciais efeitos adversos sobre a frequência e o ritmo cardíaco podem surgir após administração de xilazina, incluindo a ocorrência de bradiarritmias, como bloqueios atrioventriculares de primeiro, segundo e embora mais raro até terceiro grau (MUIR, 2007). O efeito dos diferentes protocolos anestésicos sobre os parâmetros eletrocardiográficos em macacos-prego estão apresentados na tabela 4.

Neste estudo, a média encontrada da duração das variáveis da onda $\mathrm{P}$, do intervalo $\mathrm{P}-\mathrm{R}$, complexo QRS e intervalo Q-T foram maiores em relação aos parâmetros do ECG investigados em macacos-prego contidos quimicamente com cetamina (GONDER; GARD; LOTT, 1980, LARSSON et al., 2012). A menor duração da onda $\mathrm{P}$, do intervalo P-R, complexo QRS e intervalo Q-T nos animais contidos com cetamina pode estar atribuída pelo fato dos anestésicos dissociativos causarem despolarização ventricular prematura e taquicardia sinusal (LIN, 2007, MUIR, 2007) e consequentemente reduzir a duração destes parâmetros. Em contrapartida, de acordo com o estudo de Santana et al. (2008) a associação da xilazina a cetamina resulta em bradicardia severa e consequentemente causa um prolongamento do intervalo QT em relação aos protocolos que não incluem agentes $\alpha 2$-agonistas (Tabela 4). 
Tabela 4. Valores referentes aos parâmetros eletrocardiográficos em macacos-prego (Sapajus apella) de acordo com a contenção química utilizada.

\begin{tabular}{|c|c|c|c|c|c|c|c|c|}
\hline $\begin{array}{l}\text { Protocolo } \\
\text { Anestésico }\end{array}$ & $\begin{array}{c}\text { FC } \\
\text { (bpm) }\end{array}$ & $\begin{array}{l}\text { Eixo }\left(^{\circ}\right) \\
\text { DI/DII }\end{array}$ & $\begin{array}{c}P \\
\text { duração } \\
\text { (seg) / } \\
\text { amplitude } \\
\text { (mV) }\end{array}$ & $\begin{array}{l}\text { P-R } \\
\text { (seg) }\end{array}$ & $\begin{array}{l}\text { QRS } \\
\text { duração } \\
\text { (seg)/ } \\
\text { amplitude } \\
\text { (mV) }\end{array}$ & $\begin{array}{l}\text { T } \\
\text { Pol } \\
(\%)\end{array}$ & $\begin{array}{l}\text { Q-T } \\
\text { (seg) }\end{array}$ & $\begin{array}{l}\text { ST } \\
(\%)\end{array}$ \\
\hline $\begin{array}{c}\text { Midazolam- } \\
\text { propofol } \\
\text { n:18 } \\
\text { (Fonte: } \\
\text { Elaboração } \\
\text { dos autores) }\end{array}$ & $198,4 \pm 22,9$ & $66,3 \pm 23,8$ & $\begin{array}{c}0,04 \pm 0,007 / \\
0,13 \pm 0,04\end{array}$ & $0,08 \pm 0,01$ & $\begin{array}{l}0,04 \pm 0,01 / \\
0,70 \pm 0,4\end{array}$ & $\begin{array}{l}+: 77,8 \% \\
-: 22,2 \%\end{array}$ & $\begin{array}{c}0,17 \\
\pm \\
0,03\end{array}$ & $\begin{array}{l}\text { N: } 55,6 \\
\text { ELE 0,1: } \\
27,8 \% \\
\text { DEP 0,1: } \\
16,7 \%\end{array}$ \\
\hline $\begin{array}{c}\text { Cetamina } \\
\text { n:97 } \\
\text { (LARSSON } \\
\text { et al., 2012) }\end{array}$ & $257 \pm 33$ & $\begin{array}{c}\text { Maior } \\
\text { incidência } \\
\text { do eixo } \\
\text { entre } 60- \\
90^{\circ}\end{array}$ & $\begin{array}{c}0,03 \pm 0,01 / \\
0,25 \pm 0,08\end{array}$ & $0,07 \pm 0,01$ & $\begin{array}{l}0,03 \pm 0,01 / \\
0,68 \pm 0,4\end{array}$ & $\begin{array}{l}+: 84,6 \% \\
-: 4,1 \%, \\
\text { Iso: } 7,2 \%\end{array}$ & $\begin{array}{c}0,14 \\
\pm \\
0,02\end{array}$ & $\begin{array}{lr}\text { N: } 84,6 \% \\
\text { ELE } & 0,1: \\
4,2 \% & \\
\text { ELE } & 0,2: \\
2 \% & \\
\text { DEP } & 0,1: \\
7,2 \% & \\
\text { DEP } & 0,2: \\
2 \% & \end{array}$ \\
\hline $\begin{array}{c}\text { Cetamina } \\
\mathrm{n}: 13 \\
\text { (GONDER, } \\
\text { GARD E } \\
\text { LOTT, 1980) }\end{array}$ & $230 \pm 27$ & $58 \pm 31$ & $\begin{array}{c}0,03 \pm 0,01 \\
0,35 \pm 0,12\end{array}$ & $0,08 \pm 0,01$ & $\begin{array}{l}0,02 \pm 0,01 / \\
0,9 \pm 0,3\end{array}$ & $\mathrm{Ni}$ & $\begin{array}{c}0,15 \\
\pm \\
0,01\end{array}$ & $\mathrm{Ni}$ \\
\hline $\begin{array}{c}\text { Cetamina- } \\
\text { xilazina } \\
\text { n:8 } \\
\text { (SANTANA } \\
\text { et al., 2008) }\end{array}$ & $123 \pm 19,8$ & $\mathrm{Ni}$ & $\begin{array}{c}\mathrm{Ni} / \\
0,12 \pm 0,04\end{array}$ & $0,08 \pm 0,01$ & $\begin{array}{l}0,04 \pm 0,005 / \\
0,57 \pm 0,34\end{array}$ & $\mathrm{Ni}$ & $\begin{array}{c}0,2 \\
\pm \\
0,02\end{array}$ & $\mathrm{Ni}$ \\
\hline $\begin{array}{c}\text { Tiletamina- } \\
\text { zolazepam } \\
\text { n:8 } \\
\text { (SANTANA } \\
\text { et al., 2008) }\end{array}$ & $212 \pm 23,7$ & $\mathrm{Ni}$ & $\begin{array}{c}\mathrm{Ni} / \\
0,21 \pm 0,06\end{array}$ & $0,08 \pm 0,01$ & $\begin{array}{l}0,03 \pm 0,01 / \\
0,43 \pm 0,16\end{array}$ & $\mathrm{Ni}$ & $\begin{array}{c}0,14 \\
\pm \\
0,02\end{array}$ & $\mathrm{Ni}$ \\
\hline
\end{tabular}

Siglas: ECG (eletrocardiograma); bpm (batimentos por minuto); FC (frequência cardíaca); P (onda P); P-R (intervalo PR); QRS (complexo QRS); R (onda R); T (onda T); Q-T (intervalo QT); ST (segmento ST); milivolts (mV); segundos (seg); Pol (polaridade); ELE 0,1 (elevação de 0,1mV); ELE 0,2 (elevação de 0,2mV); DEP 0,1 (depressão de 0,1mV); DEP 0,2 (depressão de 0,2mV); N (normal); S (desvio padrão); Ni (Não informado); + (Positiva); - (Negativa) e Iso (Isoelétrica).

Fonte: Elaboração dos autores.

A média da amplitude da onda P encontrada neste estudo foi intermediária aos valores encontrados em macacos anestesiados com cetamina-xilazina e tiletamina-zolazepam (SANTANA et al., 2008), mas menor em relação aos anestesiados somente com cetamina(GONDER; GARD; LOTT, 1980,LARSSON et al., 2012), (Tabela 4). A média da amplitude da onda $\mathrm{R}$ neste estudo foi similar aos macacos anestesiados com cetamina (LARSSON et al., 2012), mas maior em relação aos macacos anestesiados com cetaminaxilazina e tiletamina-zolazepam (SANTANA et al., 2008), conforme tabela 4. 
Outros parâmetros eletrocardiográficos tiveram resultados similares ao compararmos a utilização de midazolam-propofol e cetamina, não parecendo ter grandes influências nestes parâmetros, como: a onda $\mathrm{T}$ foi positiva e o segmento $\mathrm{S}$-T apresentou-se normal na maioria dos animais, assim como a maior incidência do eixo elétrico entre 60-90 (GONDER; GARD; LOTT, 1980, LARSSON et al., 2012), (Tabela 4).

A diferença nos parâmetros do ECG entre os estudos pode ter ocorrido devido a fatores individuais, mas principalmente relacionado à diferença dos protocolos anestésicos utilizados que atuam de diferentes formas sobre o sistema cardiovascular, inerentemente relacionado a fatores inotrópicos e cronotrópicos (FELIPPE, 2007; MUIR, 2007; LARSSON et al., 2012).

Primatas capturados podem receber com segurança propofol para indução e manutenção da anestesia geral, corroborando com o descrito por Flecknell, Richardson e Popovic (2007). A grande vantagem do uso do propofol em relação à cetamina em primatas não humanos é o menor tempo de recuperação anestésica e uma melhor recuperação pós-operatória (AUTHIER et al., 2006). Outras propriedades favoráveis ao seu uso são a redução do laringoespasmo durante a intubação orotraqueal, mínima náusea e mínimo movimento involuntário (FOWLER et al., 2001; BRANSON, 2007). Neste estudo a administração intramuscular de midazolam $(1 \mathrm{mg} / \mathrm{kg})$ promoveu sedação leve com relaxamento muscular. Alternativamente, a administração de midazolam $(3 \mathrm{mg} / \mathrm{kg}$ ) pode ser realizada por via oral para promover efeito calmante, reduzir o estresse e facilitar a contenção previamente à anestesia de primatas não humanos (PULLEY; ROBERTS; LERCHE, 2004).

\section{Conclusões}

A administração intramuscular de midazolam, seguida de anestesia geral de curta ação com propofol não causou arritmias no traçado eletrocardiográfico e preservou a FC nos limites considerados fisiológicos para a espécie. Consequentemente, foi observada uma duração mais longa do intervalo Q-T comparado aos estudos anteriores que utilizaram somente cetamina.

\section{Referências}

AUTHIER, S.; CHAURAND, F.; LEGASPIM, M.; BREAULT, C.; TRONCY, E. Comparison of three anesthetic protocols for intraduodenal drug administration using endoscopy in rhesus monkeys (Macaca mulatta). Journal of the American Association for Laboratory Animal Science, Germany, v. 45, n. 6, p. 73-79, 2006.

BRANSON, K. R. Injectable and alternative anesthetic techniques. In: TRANQUILLI, W. J.; THURMON, J. C.; GRIMM, K. A. (Ed.). Lumb \& jones' veterinary anesthesia and analgesia. $4^{\text {th }}$ ed. Blackwell Publishing: Iowa, 2007. p. 291-292.

BRODBELT, D. C.; BLISSITT, K. J.; HAMMOND, R. A.; NEATH, P. J.; YOUNG, L. E.; PFEIFFER, D. U.; WOOD, J. L. The risk of death: the confidential enquiry into perioperative small animal fatalities. Veterinary Anaesthesia and Analgesia, Malden, v. 35, n. 5, p. 365-373, 2008. Disponível em: $\quad<$ http://onlinelibrary.wiley.com/doi/10.1111/ j.1467-2995.2008.00397.x/abstract; jsessionid= 1F6751D6B72722E02A52F0583355D13A.d03t01>. Acesso em: 20 nov. 2012.

FALASCA, C. A.; GRANA, D. R.; MARESO, E. A.; GOMEZ, E.; GILI, M. M. Electrocardiographic changes in chronic Trypanosoma cruzi infected Sapajus apella monkeys. Arquivos Brasileiros de Cardiologia, Rio de Janeiro, v. 56, n. 4, p. 287-293, 1991.

FELIPPE, P. A. N. Eletrocardiografia. In: CUBAS, Z. S.; SILVA, J. C. R.; CATÃO-DIAS, J. L. (Ed.). Tratado de animais selvagens medicina veterinária. Roca: São Paulo, 2007. p. 920-929.

FLECKNELL, P. A.; RICHARDSON, C. A.; POPOVIC, A. Laboratory Animals. In: TRANQUILLI, W. J.; THURMON, J. C.; GRIMM, K. A. (Ed.). Lumb \& jones' veterinary anesthesia and analgesia. $4^{\text {th }}$ ed. Blackwell Publishing: Iowa, 2007. p. 776-778.

FOWLER, K. A.; HUERKAMP, M. J.; PULLIUM, J. K.; SUBRAMANIAN, T. Anesthetic protocol: propofol use in Rhesus macaques (Macaca mulatta) during magnetic resonance imaging with stereotactic head frame application. Brain Research Protocols, Amsterdam, v. 7, n. 2, p. 87-93, 2001. Disponível em: 
$<$ http://www.sciencedirect.com/science/article/pii/ S1385299X00000519>. Acesso em: 14 set. 2012.

GONDER, J. C.; GARD, E. A.; LOTT, N. E. 3rd. Electrocardiograms of nine species of nonhuman primate sedated with ketamine. Americav Journal of Veterinary Research, Schaumburg, v. 41, n. 6, p. 972-975, 1980.

LARSSON, M. H. M. A.; LUCAS, S. R. R.; MIRANDOLA, R. M. S.; LAZARETTI, P.; FEDULHO, J. D. L.; GUIMARÃES, M. A. B. V. Valores de referencia das provas de funçoes hepática, renal e de alguns eletrólitos em Sapajus apella, anestesiados com cetamina. Ciência Rural, Santa Maria,v.27,n.2,p.257-262,1997.Disponível em: <http://www.scielo.br/scielo.php?script=sci_arttext $\&$ pid $=$ S0103-84781997000200014 $>$. Acesso em: 13 nov. 2012.

LARSSON, M.H.M.A.; PELLEGRINO,A.; OLIVEIRA, V. M.; PRADA, C. S.; FEDULHO, J. D., LARSSON JUNIOR, C. E. Electrocardiographic parameters of captive tufted capuchins (Sapajus apella) under chemical immobilization. Journal of Zoo and Wildlife Medicine, Yulle, v. 43, n. 4, p. 715-718, 2012. Disponível em: $<$ http://dx.doi.org/10.1638/2010-0061R1.1>. Acesso em: 12 jan. 2013.

LARSSON, M. H. M. A.; BIRGEL, E. H.; BENESI, F. J.; BIRGEL JUNIOR, E. H.; LAZARETTI, P.; FEDULLO, J. D. L.; LARSSON JUNIOR, C. E.; MOLINA, S. R.; GUERRA, P. P. C. A.; PRADA, C. S. Hematological values of Sapajus apella anesthetized with ketamine. Brazilian Journal of Veterinary Research and Animal Science, Saõ Paulo, v. 36, n. 3, p. 131-135, 1999. Disponível em: <http://www.revistas.usp.br/bjvras/ article/view/5756/49903>. Acesso em: 22 nov. 2012.

LEMKE, K. A. Anticholinergics and sedatives. In: TRANQUILLI, W. J.; THURMON, J. C.; GRIMM, K. A. (Ed.). Lumb \& jones' veterinary anesthesia and analgesia. $4^{\text {th }}$ ed. Blackwell Publishing: Iowa, 2007. p. 229-230.

LIN, H. Dissociative Anesthetic. In: TRANQUILLI, W. J.; THURMON, J. C.; GRIMM, K. A. (Ed.). Lumb $\&$ jones' veterinary anesthesia and analgesia. $4^{\text {th }}$ ed. Blackwell Publishing: Iowa, 2007. p. 302-303.
MUIR, W. W. Cardiovascular system. In: TRANQUILLI, W. J.; THURMON, J. C.; GRIMM, K. A. (Ed.). Lumb $\&$ jones' veterinary anesthesia and analgesia. $4^{\text {th }} \mathrm{ed}$. Blackwell Publishing: Iowa, 2007. p. 83-112.

PULlEY, A. C.; ROBERTS, J. A.; LERCHE, N. W. Four preanesthetic oral sedation protocols for rhesus macaques (Macaca mulatta). Journal of Zoo and Wildlife Medicine, Yulle, v. 35, n. 4, p. 497-502, 2004. Disponível em: <http://www.bioone.org/doi/full/10.1638/03-092>. Acesso em: 15 nov. 2011.

SANTANA, V. L.; SILVA, R. M. N.; SOUZA, A. P.; FERREIRA, A. F.; WAGNER, P. G. C.; EVÊNCIO, J.; NÓBREGA, P. I. Estudo comparativo dos efeitos da associação anestésica cetamina-xilazina ou tiletaminazolazepam em macacos-prego (Sapajus apella - Linnaeus, 1758). Revista Científica de Medicina VeterináriaPequenos Animais e Animais de Estimação, Curitiba, v. 6, n. 8, p. 159-165, 2008.

SUAREZ, M. A.; DZIKITI, B. T.; STEGMANN, F. G.; HARTMAN, M. Comparison of alfaxalone and propofol administered as total intravenous anaesthesia for ovariohysterectomy in dogs. Veterinary Anaesthesia and Analgesia, Malden, v. 39, n. 3, p. 236-244, 2012. Disponível em: <http://www. onlinelibrary.wiley.com/ doi/10.1111/j.1467-2995.2011.00700.x/pdf $>$. Acesso em: 11 jan. 2013.

VERONA, C. E. S.; PISSINATTI, A. Primates- primatas do novo mundo (Sagui, Macaco-prego, Macaco-aranha, Bugio). In: CUBAS, Z. S.; SILVA, J. C. R.; CATÃODIAS, J. L. (Ed.). Tratado de animais selvagens medicina veterinária. Roca: São Paulo, 2007. p. 358-362.

YOO, Y.; BAI, S. J.; LEE, K. Y.; SHIN, S.; CHOI, E. K.; LEE, J. W. Total Intravenous anesthesia with propofol reduces postoperative nausea and vomiting in patients undergoing robot-assisted laparoscopic radical prostatectomy: a prospective randomized trial. Yonsei Medical Journal, Korea, v. 53, n. 6, p. 1197-1202, 2012. Disponível em: <http://www.eymj.org/DOIx. php?id=10.3349/ymj.2012.53.6.1197>. Acesso em: 11 jan. 2013. 